EPJ Web of Conferences 45, 01054 (2013)

DOI: $10.1051 /$ epjconf/20134501054

(C) Owned by the authors, published by EDP Sciences, 2013

\title{
NUMERICAL SIMULATION OF THE FLOW AROUND DIFFUSIBLE BARRIERS
}

\author{
Martin Kyncl $^{1, \mathrm{a}}$ and Jaroslav Pelant ${ }^{1, \mathrm{~b}}$ \\ ${ }^{1}$ Výzkumný a Zkušební Letecký Ústav, VZLÚ, \\ Beranových 130, 19905 Praha - Letňany, Czech Republic
}

\begin{abstract}
We work with the system of equations describing the non-stationary compressible fluid flow, and we focus on the numerical solution of these equations, and on the boundary conditions. In order to construct the boundary conditions we analyze the equations in the close vicinity of the boundary. Many boundary conditions (i.e. fixed, linearized) do not solve the local problem in an appropriate way, which may bring non-physical errors into the solution, slow down the convergent process, or even ruin the solution in the whole domain. We use the analysis of the exact solution of the Riemann problem in order to solve this local boundary problem. In the case of the diffusible barrier we solve the modified Riemann problem, with the one-side initial condition, complemented with the Darcy's law and added inertial loss. We analyze the solution of this problem. We show the computational results of the flow through the diffusible barrier, obtained with the own-developed code for the solution of the compressible gas flow.
\end{abstract}

\section{Introduction}

The physical theory of the compressible fluid motion is based on the principles of conservation laws of mass, momentum, and energy. The mathematical equations describing these fundamental conservation laws form a system of partial differential equations (the Euler Equations, the Navier-Stokes Equations, the Navier Stokes Equations with turbulent models). We focus on the numerical solution of these equations. The correct design of the boundary conditions plays also an important role in the numerical modeling of the processes involved. We choose the well-known finite volume method to discretize the analytical problem, represented by the system of the equations in generalized (integral) form. To apply this method we split the area of the interest into the elements, and we construct a piecewise constant solution in time. The crucial problem of this method lies in the evaluation of the so-called fluxes through the edges/faces of the particular elements. In order to compute these fluxes, various methods can be used. One of the most accurate method (and perhaps the most accurate method) is based on the solution of the so-called Riemann problem for the 2D/3D split Euler equations. Unfortunately, the exact solution of this problem cannot be expressed in a closed form, and has to be computed by an iterative process (to given accuracy). Therefore this method is also one of the most demanding. Nevertheless, on account of the accuracy of the Riemann solver, we decided to use the analysis of the exact solution also for the discretization of the fluxes through the boundary edges/faces and on the edges/faces simulating the diffusible barrier. The incomplete local Riemann problem at the diffusible barrier is complemented with the Darcy's law and added inertial loss. In this work we analyze the modified local problem. We construct own algorithm for the solution of the boundary problem at the

\footnotetext{
a e-mail: kyncl@vzlu.cz

b e-mail: pelant@vzlu.cz
}

diffusible barrier, and we use it in the numerical examples.

\section{The Navier-Stokes Equations}

Here we show the so-called Navier-Stokes equations. We consider the conservation laws for viscous compressible flow with the zero outer volume forces and heat sources in a domain $\Omega \in R^{N}$, and time interval $(0, T)$, with $T>0$. Using the notation in [2, page 376], we write

$$
\frac{\partial \boldsymbol{w}}{\partial t}+\sum_{s=1}^{N} \frac{\partial \boldsymbol{f}_{s}(\boldsymbol{w})}{\partial x_{s}}=\sum_{s=1}^{N} \frac{\partial \boldsymbol{R}_{s}(\boldsymbol{w}, \nabla \boldsymbol{w})}{\partial x_{s}} \quad \text { in } Q_{T}=\Omega \times(0, T) \text {. }
$$

Here $N$ denotes the dimension $(N=2$ or 3 for $2 \mathrm{D}$ or $3 \mathrm{D}$ flow, respectively), $\boldsymbol{w}=\boldsymbol{w}(x, t)$ is the state vector, $x \in \Omega, t$ denotes the time, $Q_{T}$ is called a space-time cylinder, $\boldsymbol{f}_{s}$ are the inviscid (Euler) fluxes, $\boldsymbol{R}_{S}$ are viscous fluxes:

$$
\begin{array}{r}
\boldsymbol{w}=\left(w_{1}, w_{2}, \ldots, w_{N+2}\right)^{T}=\left(\varrho, \varrho v_{1}, \ldots, \varrho v_{N}, E\right)^{T}, \\
\boldsymbol{f}_{s}(\boldsymbol{w})=\left(\varrho v_{s}, \varrho v_{s} v_{1}+\delta_{s 1} p, \ldots, \varrho v_{s} v_{N}+\delta_{s N} p,(E+p) v_{s}\right)^{T} \\
\boldsymbol{R}_{s}(\boldsymbol{w}, \nabla \boldsymbol{w})=\left(0, \tau_{s 1}, \ldots, \tau_{s N}, \sum_{r=1}^{N} \tau_{s r} v_{r}+k \partial \theta / \partial x_{s}\right)^{T}, \\
\tau_{i j}=\lambda \operatorname{div} \boldsymbol{v} \delta_{i j}+2 \mu d_{i j}(\boldsymbol{v}), \quad d_{i j}(\boldsymbol{v})=\frac{1}{2}\left(\frac{\partial v_{i}}{\partial x_{j}}+\frac{\partial v_{j}}{\partial x_{i}}\right),
\end{array}
$$

with $\boldsymbol{v}=\left(v_{1}, \ldots, v_{N}\right)^{T}$ denoting the velocity vector, $\varrho$ being the density, $p$ the pressure, $\theta$ the absolute temperature, $E$ the total energy, $k$ the heat conduction coefficient, and $\mu, \lambda$ denoting the viscosity coefficients. In this work we assume the relation $3 \lambda+2 \mu=0$ is valid. We add the thermodynamical relations to the system (1)

$$
p=(\gamma-1)\left(E-\varrho v^{2} / 2\right), \quad \theta=\left(\frac{E}{\varrho}-\frac{1}{2} v^{2}\right) / c_{v},
$$


with the $\gamma$ denoting the Poisson adiabatic constant and $c_{v}$ the specific heat at constant volume. Within the numerical solution of this problem we usually consider the equations in the more general integral form, which allows the discontinuities in the solution. It is well known that the classical Navier-Stokes equation for three-dimensional incompressible viscous flow is invariant under the Galilean transformations, see [1, page 69]. We can choose a new Cartesian coordinate system $\left(\tilde{x}_{1}, \tilde{x}_{2}, \tilde{x}_{3}\right)$ such that the origin is at the point $\tilde{\sigma}$, and the axis $\tilde{x}_{1}$ is in the direction of a chosen unit vector $\boldsymbol{n}=\left(n_{1}, n_{2}, n_{3}\right),|\boldsymbol{n}|=1$. This transformation can be written

$$
\left(\begin{array}{l}
\tilde{x}_{1} \\
\tilde{x}_{2} \\
\tilde{x}_{3}
\end{array}\right)=\mathbb{Q}_{0}\left(\begin{array}{l}
x_{1} \\
x_{2} \\
x_{3}
\end{array}\right)+\tilde{\sigma}, \text { with } \mathbb{Q}_{0}=\left(\begin{array}{l}
\boldsymbol{n} \\
\boldsymbol{o} \\
\boldsymbol{p}
\end{array}\right)=\left(\begin{array}{lll}
n_{1} & n_{2} & n_{3} \\
o_{1} & o_{2} & o_{3} \\
p_{1} & p_{2} & p_{3}
\end{array}\right) \text {. }
$$

Here $\mathbb{Q}_{0}$ is the rotation matrix, the elements of the rotation matrix are not all independent. The vectors $\boldsymbol{n}, \boldsymbol{o}, \boldsymbol{p}$ must have the properties

$$
|\boldsymbol{n}|=|\boldsymbol{o}|=1, \quad \boldsymbol{n} \cdot \boldsymbol{o}=0, \quad \boldsymbol{p}=\boldsymbol{n} \times \boldsymbol{o} .
$$

We can choose the vector $\boldsymbol{o}=\left(o_{1}, o_{2}, o_{3}\right)$ such that $\boldsymbol{n} \cdot \boldsymbol{o}=$ $0,|\boldsymbol{o}|=1$. And then the vector $\boldsymbol{p}=\left(p_{1}, p_{2}, p_{3}\right)$ is determined by $\boldsymbol{p}=\boldsymbol{n} \times \boldsymbol{o}$. The axis $\tilde{x}_{2}$ points in the direction of the vector $\boldsymbol{o}$, and the axis $\tilde{x}_{3}$ in the direction of the vector $\boldsymbol{p}$. The matrix $\mathbb{Q}_{0}$ is orthogonal, and the vectors are orthogonal unit vectors. The inverse of the orthogonal matrix $\mathbb{Q}_{0}$ is $\mathbb{Q}_{0}^{-1}=\mathbb{Q}_{0}^{T}$. The transformation of the state vector $\boldsymbol{w}$ yields the state vector $\boldsymbol{q}$ in the new coordinates. It is

$$
\begin{aligned}
& \boldsymbol{q}=\mathbb{Q} \boldsymbol{w}, \\
& \boldsymbol{w}=\mathbb{Q}^{-1} \boldsymbol{q},
\end{aligned}
$$

with

$$
\mathbb{Q}=\left(\begin{array}{ccccc}
1 & 0 & 0 & 0 & 0 \\
0 & n_{1} & n_{2} & n_{3} & 0 \\
0 & o_{1} & o_{2} & o_{3} & 0 \\
0 & p_{1} & p_{2} & p_{3} & 0 \\
0 & 0 & 0 & 0 & 1
\end{array}\right), \quad \mathbb{Q}^{-1}=\left(\begin{array}{ccccc}
1 & 0 & 0 & 0 & 0 \\
0 & n_{1} & o_{1} & p_{1} & 0 \\
0 & n_{2} & o_{2} & p_{2} & 0 \\
0 & n_{3} & o_{3} & p_{3} & 0 \\
0 & 0 & 0 & 0 & 1
\end{array}\right) .
$$

Using this transformation of the coordinates, the NavierStokes equations keep the form

$$
\frac{\partial \boldsymbol{q}}{\partial t}+\sum_{s=1}^{3} \frac{\partial \boldsymbol{f}_{s}(\boldsymbol{q})}{\partial \tilde{x}_{s}}=\sum_{s=1}^{N} \frac{\partial \boldsymbol{R}_{s}(\boldsymbol{q}, \nabla \boldsymbol{q})}{\partial \tilde{x}_{s}}
$$

\section{The Euler Equations}

Ommiting the vicsous terms in (1) we get the system of the Euler equations

$$
\frac{\partial \boldsymbol{w}}{\partial t}+\sum_{s=1}^{N} \frac{\partial \boldsymbol{f}_{s}(\boldsymbol{w})}{\partial x_{s}}=0 \quad \text { in } Q_{T}=\Omega \times(0, T) .
$$

The system of the Euler equations is rotationally invariant (see e.g. [2, page 108]).

\section{FVM Discretization of the Problem}

Here we describe the so-called finite volume discretization of the problem (1) in the domain $\Omega$. By $\Omega_{h}$ let us the denote the polyhedral approximation of $\Omega$. The system of the closed polyhedrons with mutually disjoint interiors $\mathcal{D}_{h}=\left\{D_{i}\right\}_{i \in J}$, where $J \subset Z^{+}=\{0,1, \ldots\}$ is an index set and $h>0$, will be called a finite volume mesh. This system $\mathcal{D}_{h}$ approximates the domain $\Omega$, we write $\bar{\Omega}_{h}=\bigcup_{i \in J} D_{i}$. The elements $D_{i} \in \mathcal{D}_{h}$ are called the finite volumes. For two neighboring elements $D_{i}, D_{j}$ we set $\Gamma_{i j}=\partial D_{i} \cap \partial D_{j}=\Gamma_{j i}$. Here we will work with the so-called regular meshes, i.e. the intersection of two arbitrary (different) elements is either empty or it consists of a common vertex or a common edge or a common face (in 3D). The boundary $\partial D_{i}$ of each element $D_{i}$ is $\partial D_{i}=\bigcup_{\Gamma_{i j} \in \Gamma_{D_{i}}} \Gamma_{i j}$. Here the set $\Gamma_{D_{i}}=\left\{\Gamma_{i j} ; \Gamma_{i j} \subset \partial D_{i}\right\}$ forms the boundary $\partial D_{i}$. By $\boldsymbol{n}_{i j}$ let us denote the unit outer normal to $\partial D_{i}$ on $\Gamma_{i j}$. Let us construct a partition $0=t_{0}<t_{1}<\ldots$ of the time interval $[0, T]$ and denote the time steps $\tau_{k}=t_{k+1}-t_{k}$. We integrate the system (1) over the set $D_{i} \times\left(t_{k}, t_{k+1}\right)$, and we use the Green's theorem on $D_{i}$. With the integral form of the equations we can study a flow with discontinuities, such as shock waves, too. It is

$$
\begin{array}{r}
\int_{D_{i}}\left(\boldsymbol{w}\left(x, t_{k+1}\right)-\boldsymbol{w}\left(x, t_{k}\right)\right) d x+ \\
+\int_{t_{k}}^{t_{k+1}} \sum_{\Gamma_{i j} \in \Gamma_{D_{i}}} \int_{\Gamma_{i j}} \sum_{s=1}^{3} \boldsymbol{f}_{s}(\boldsymbol{w})\left(n_{i j}\right)_{s} d S d t \\
=\int_{t_{k}}^{t_{k+1}} \sum_{\Gamma_{i j} \in \Gamma_{D_{i}}} \int_{\Gamma_{i j}} \sum_{S=1}^{3} \boldsymbol{R}_{s}(\boldsymbol{w}, \nabla \boldsymbol{w})\left(n_{i j}\right)_{S} d S d t .
\end{array}
$$

We define a finite volume approximate solution of the system studied (1) as a piecewise constant vector-valued functions $\mathbf{w}_{h}^{k}, k=0,1, \ldots$, where $\boldsymbol{w}_{h}^{k}$ is constant on each element $D_{i}$, and $t_{k}$ is the time instant. By $\boldsymbol{w}_{i}^{k}$ we denote the value of the approximate solution on $D_{i}$ at time $t_{k}$. We approximate the integral over the element $D_{i}$

$$
\int_{D_{i}} \boldsymbol{w}\left(x, t_{k}\right) d x \approx\left|D_{i}\right| \boldsymbol{w}_{i}^{k} .
$$

Further we proceed with the approximation of the fluxes. Usually the flux $\left.\sum_{s=1}^{3} \boldsymbol{f}_{s}(\boldsymbol{w})\left(n_{i j}\right)_{s}\right|_{\Gamma_{i j}}$ is being approximated by a numerical flux $\boldsymbol{H}\left(\boldsymbol{w}_{i}^{l}, \boldsymbol{w}_{j}^{l}, \boldsymbol{n}_{i j}\right)$ at suitable time instant $t_{l}$. Here we show the numerical flux based on the solution of the Riemann problem for the split Euler equations. By $\boldsymbol{w}_{\Gamma_{i j}}^{l}$ let us denote the state vector $\boldsymbol{w}$ at the center of the edge $\Gamma_{i j}$ at the time instant $t_{l}$, and let us suppose $\boldsymbol{w}_{\Gamma_{i j}}^{l}$ is known. Evaluation of these values will be a question of the further analysis, here we use them to approximate the integrals in (5) with the one-point rule. It is possible to approximate the system (5) by the following explicit finite volume scheme

$$
\begin{aligned}
\left|D_{i}\right|\left(\boldsymbol{w}_{i}^{k+1}-\boldsymbol{w}_{i}^{k}\right)+\tau_{k} \sum_{\Gamma_{i j} \in \Gamma_{D_{i}}}\left|\Gamma_{i j}\right| \sum_{s=1}^{3} \boldsymbol{f}_{s}\left(\boldsymbol{w}_{\Gamma_{i j}}^{k}\right) n_{s} \\
=\tau_{k} \sum_{\Gamma_{i j} \in \Gamma_{D_{i}}}\left|\Gamma_{i j}\right| \sum_{s=1}^{3} \boldsymbol{R}_{s}\left(\boldsymbol{w}_{\Gamma_{i j}}^{k}, \nabla \boldsymbol{w}_{\Gamma_{i j}}^{k}\right) n_{s} .
\end{aligned}
$$

Here $\nabla \boldsymbol{w}_{\Gamma_{i j}}^{l}$ denotes the $\nabla \boldsymbol{w}$ at the center of the edge $\Gamma_{i j}$ at time instant $t_{l}$. With this finite volume formula one computes the values of the approximate solution at the time instant $t_{k+1}$, using the values from the time instant $t_{k}$, and 
by evaluating the values $\boldsymbol{w}_{\Gamma_{i j}}^{k}$ at the faces $\Gamma_{i j}$. In order to achieve the stability of the used method, the time step $\tau_{k}$ must be restricted by the so-called CFL condition, see [2]. The approximation of the values $\nabla \boldsymbol{w}_{\Gamma_{i j}}^{l}$ can be done via the dual mesh, see [3]. The crucial problem of our work lies with the evaluation of the edge values $w_{\Gamma_{i j}}^{k}$.

\section{Inner Faces}

To approximate the values $\boldsymbol{w}_{\Gamma_{i j}}^{k}$ at the inner faces/edges we solve the simplified system (8) in the vicinity of the face $\Gamma_{i j}$ in time with the initial condition formed by the state vectors $\boldsymbol{w}_{i}^{k}$ and $\boldsymbol{w}_{j}^{k}$. Using the rotational invariance of the Euler equations, the system (4) is expressed in a new Cartesian coordinate system $\tilde{x}_{1}, \tilde{x}_{2}, \tilde{x}_{3}$ with the origin at the center of the gravity of $\Gamma_{i j}$ and with the new axis $\tilde{x}_{1}$ in the direction of $\boldsymbol{n}=\left(n_{1}, n_{2}, n_{3}\right)$, given by the face normal $\boldsymbol{n}=\boldsymbol{n}_{i j}$.

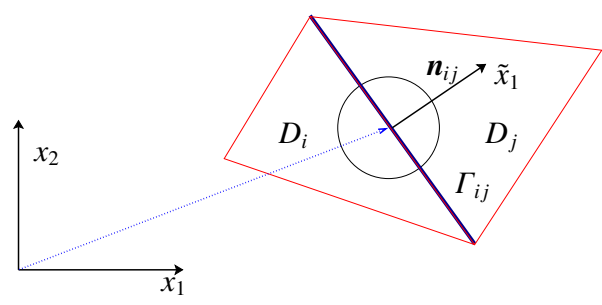

Fig. 1. Coordinate transformation for the inner edges in 2D.

Then the derivatives with respect to $\tilde{x}_{2}, \tilde{x}_{3}$ are neglected and we get the so-called split 3D Euler equations, see [2, page 138]:

$$
\frac{\partial \boldsymbol{q}}{\partial t}+\frac{\partial \boldsymbol{f}_{1}(\boldsymbol{q})}{\partial \tilde{x}_{1}}=0
$$

The values $\boldsymbol{w}_{i}^{k}$ and $\boldsymbol{w}_{j}^{k}$ adjacent to the face $\Gamma_{i j}$ are known, forming the initial conditions

$$
\begin{array}{ll}
\boldsymbol{q}\left(\tilde{x}_{1}, 0\right)=\boldsymbol{q}_{L}=\mathbb{Q} \boldsymbol{w}_{i}^{k}, & \tilde{x}_{1}<0, \\
\boldsymbol{q}\left(\tilde{x}_{1}, 0\right)=\boldsymbol{q}_{R}=\mathbb{Q} \boldsymbol{w}_{j}^{k}, & \tilde{x}_{1}>0 .
\end{array}
$$

The transformation matrix $\mathbb{Q}$ is defined in (3). In this work we will refer to these initial conditions as to the left-hand side initial condition (9) and the right-hand side initial condition (10). The problem (8), (9), (10) has a unique "solution" in $(-\infty, \infty) \times(0, \infty)$, the analysis can be found in $[2$, page 138]. The solution of this problem is fundamentally the same as the solution of the Riemann problem for the 1D Euler equations, see [2, page 138]. In fact, the solution for the pressure, the first component of the velocity (in the direction of the axis $\tilde{x}_{1}$ ), and the density is exactly the same as in one-dimensional case. The remaining components of the velocity change only across the middle wave (described in Section 4) in general. Let $\boldsymbol{q}_{R S}\left(\boldsymbol{q}_{L}, \boldsymbol{q}_{R}, \tilde{x}_{1}, t\right)$ denote the solution of the problem (8), (9), (10) at the point $\left(\tilde{x}_{1}, t\right)$. We are interested in the solution of this local problem at the time axis, which is the sought solution in the local coordinates $\boldsymbol{q}_{\Gamma_{i j}}=\boldsymbol{q}_{R S}\left(\boldsymbol{q}_{L}, \boldsymbol{q}_{R}, 0, t\right)$. The backward transformation (3) of the state vector $\boldsymbol{q}_{\Gamma_{i j}}$ into the global coordinates is

$$
\boldsymbol{w}_{\Gamma_{i j}}^{k}=\mathbb{Q}^{-1} \boldsymbol{q}_{\Gamma_{i j}}=\mathbb{Q}^{-1} \boldsymbol{q}_{R S}\left(\mathbb{Q} \boldsymbol{w}_{i}^{k}, \mathbb{Q} \boldsymbol{w}_{j}^{k}, 0, t\right) .
$$

The described process of finding the face values $w_{\Gamma_{i j}}^{k}$ is independent on the choice of the vectors $\boldsymbol{o}, \boldsymbol{p}$, determining the transformation matrix $\mathbb{Q}$ defined in (3), see [3].

\section{Boundary Faces}

Let $\Gamma_{i j}$ be the face of the element $D_{i}$ laying at the boundary of the computational area. To approximate the face values $\boldsymbol{w}_{\Gamma_{i j}}^{k}$ at time instant $t_{k}$ we solve the incomplete simplified system (8) in the vicinity of the face $\Gamma_{i j}$ in time with the initial condition (9) determined by the state vector $\boldsymbol{w}_{i}^{k}$. Similarily to the situation at the inner face we use the rotational invariance of the Euler equations, and we express the system (4) in a new Cartesian coordinate system $\tilde{x}_{1}, \tilde{x}_{2}, \tilde{x}_{3}$ with the origin at the center of gravity of $\Gamma_{i j}$ and with the new axis $\tilde{x}_{1}$ in the direction of vector $\boldsymbol{n}=\left(n_{1}, n_{2}, n_{3}\right)$. The vector $\boldsymbol{n}$ corresponds to the face normal vector $\boldsymbol{n}_{i j}$ pointing out of the volume $D_{i}$, however for certain boundary conditions it is convenient to define $\boldsymbol{n}$ in a different way (still pointing out of the volume $D_{i}$ ). Then the derivatives

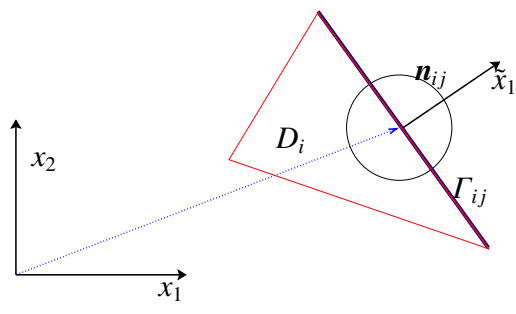

Fig. 2. Coordinate transformation for the boundary edge in 2D.

with respect to $\tilde{x}_{2}, \tilde{x}_{3}$ are neglected and we get the so-called split 3D Euler equations (8). The state vector $\boldsymbol{w}_{i}^{k}$ on the element $D_{i}$ adjacent to the face $\Gamma_{i j}$ is known, forming the initial condition for the local problem (8),(9). We seek the boundary state $\boldsymbol{q}_{\Gamma_{i j}}=\boldsymbol{q}(0, t)$. This problem is not uniquely solvable and it must be further specified with some chosen complementary conditions, see [3]. The boundary state $\boldsymbol{q}_{B}$ is then found as a solution of the problem (8),(9) and the complementary conditions. In order to construct such complementary conditions we use the analysis of the 1D Riemann problem in Section 4, see [6], [3]. For example, it is possible to prefer the given pressure, or the given velocity, or the given temperature, or the total quantities and the direction of the velocity, or the given mass flow at the boundary. In this work we focus on the boundary condition at the diffusible barrier. Section 5 shows the solution of this problem for the pressure, density, and the first component of velocity at the barrier (in local coordinates), the other components of velocity are chosen from the known initial condition. Once the local state vector $\boldsymbol{q}_{\Gamma_{i j}}$ is known, we use transformation (3) to evaluate the face state vector $\boldsymbol{w}_{\Gamma_{i j}}^{k}$ in global coordinates, it is $\boldsymbol{w}_{\Gamma_{i j}}^{k}=\mathbb{Q}^{-1} \boldsymbol{q}_{\Gamma_{i j}}$.

\section{1D Riemann Problem for the Euler Equations}

We are concerned with one-dimensional Euler equations

$$
\frac{\partial \boldsymbol{q}}{\partial t}+\frac{\partial \boldsymbol{f}(\boldsymbol{q})}{\partial \tilde{x}_{1}}=0, \quad \text { in } Q_{\infty}=(-\infty,+\infty) \times(0,+\infty)
$$

with the notation $\boldsymbol{q}=(\varrho, \varrho u, E)^{\mathrm{T}}, \quad \boldsymbol{f}(\boldsymbol{q})=\left(\varrho u, \varrho u^{2}+\right.$ $p,(E+p) u)^{\mathrm{T}}, u$ denotes the velocity, $\varrho$ the density, $p$ the 
pressure, $E$ denotes the total energy. We assume that the equation of state for ideal gas holds $p=(\gamma-1)(E-$ $\left.\varrho u^{2} / 2\right)$ in $Q_{\infty}$. Here $\gamma$ is the Poisson adiabatic constant.

The system is hyperbolic. The Riemann problem for hyperbolic system (12) consists of finding its entropy weak solution in $Q_{\infty}$, which satisfies the initial condition formed by two constant states $\boldsymbol{q}_{L}, \boldsymbol{q}_{R}$ :

$$
\begin{array}{ll}
\boldsymbol{q}\left(\tilde{x}_{1}, 0\right)=\boldsymbol{q}_{L}, & \tilde{x}_{1}<0, \\
\boldsymbol{q}\left(\tilde{x}_{1}, 0\right)=\boldsymbol{q}_{R}, & \tilde{x}_{1}>0,
\end{array}
$$

Here $\boldsymbol{q}_{L}=\left(\varrho_{L}, \varrho_{L} u_{L}, E_{L}\right)^{\mathrm{T}}, \boldsymbol{q}_{R}=\left(\varrho_{R}, \varrho_{R} u_{R}, E_{R}\right)^{\mathrm{T}}$ are known states. The physical analogue to this problem is so-called shock-tube problem. It is more convenient to use the vector of primitive variables $(\varrho, u, p)$ rather then the vector of conservative variables $(\varrho, \varrho u, E)$ in solving the Riemann problem. The general theorem on the solvability of the Riemann problem can be found in [2, page 88]. The solution $\boldsymbol{q}=\boldsymbol{q}\left(\tilde{x}_{1}, t\right)$ of the Riemann problem (12),(13),(14) is piecewise smooth and its general form can be seen from Fig. 3, where a system of half lines is drawn. These half

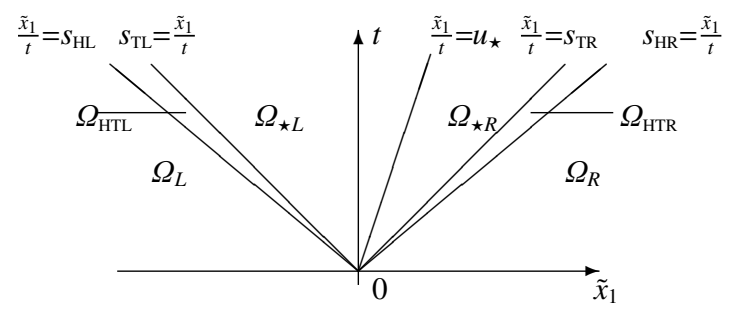

Fig. 3. Structure of the solution of the Riemann problem (12),(13),(14)

lines define regions, where $\boldsymbol{q}$ is either constant or changes smoothly. Let us define the open sets called wedges (see Fig. 3):

$$
\begin{aligned}
& \Omega_{L}=\operatorname{wedge}\left(L_{-\infty}, s_{\mathrm{HL}}\right), \\
& \Omega_{\mathrm{HTL}}=\operatorname{wedge}\left(s_{\mathrm{HL}}, s_{\mathrm{TL}}\right) \text {, } \\
& \Omega_{\star L}=\operatorname{wedge}\left(s_{\mathrm{TL}}, u_{*}\right) \text {, } \\
& \Omega_{\star R}=\operatorname{wedge}\left(u_{\star}, s_{\mathrm{TR}}\right), \\
& \Omega_{\mathrm{HTR}}=\operatorname{wedge}\left(s_{\mathrm{TR}}, s_{\mathrm{HR}}\right) \text {, } \\
& \Omega_{R}=\operatorname{wedge}\left(s_{\mathrm{HR}}, L_{+\infty}\right) \text {. }
\end{aligned}
$$

The solution in $\Omega_{L}, \Omega_{\star L}, \Omega_{\star R}, \Omega_{R}$ is constant (see e.g. [2, page 128])

$$
\left.\boldsymbol{q}\right|_{\Omega_{L}}=\boldsymbol{q}_{L},\left.\quad \boldsymbol{q}\right|_{\Omega_{\star L}}=\boldsymbol{q}_{\star L},\left.\quad \boldsymbol{q}\right|_{\Omega_{\star R}}=\boldsymbol{q}_{\star R},\left.\quad \boldsymbol{q}\right|_{\Omega_{R}}=\boldsymbol{q}_{R} .
$$

Exact solution of the Riemann problem has three waves in general. The wedges $\Omega_{L}$ and $\Omega_{\star L}$ are separated by left wave (either 1-shock wave, or 1-rarefaction wave). There is a contact discontinuity in between regions $\Omega_{\star L}$ and $\Omega_{\star R}$, it is formed by the half line $\left\{\left(\tilde{x}_{1}, t\right) ; \frac{\tilde{x}_{1}}{t}=u_{\star} ; t>0\right\}$. Wedges $\Omega_{\star R}$ and $\Omega_{R}$ are separated by right wave (either 3-shock wave, or 3-rarefaction wave). For special state $\boldsymbol{q}_{R}$ there is no right wave and it is $\boldsymbol{q}_{R}=\boldsymbol{q}_{\star R}$. The pressure $p$ and the velocity $u$ don't change across the contact discontinuity, while the density $\varrho$ changes across this discontinuity in general. We can denote the vectors of primitive variables in particular regions as

$$
\begin{aligned}
& \left.(\varrho, u, p)\right|_{\Omega_{L}}=\left(\varrho_{L}, u_{L}, p_{L}\right),\left.\quad(\varrho, u, p)\right|_{\Omega_{\star R}}=\left(\varrho_{\star R}, u_{\star}, p_{\star}\right), \\
& \left.(\varrho, u, p)\right|_{\Omega_{\star L}}=\left(\varrho_{\star L}, u_{\star}, p_{\star}\right),\left.\quad(\varrho, u, p)\right|_{\Omega_{R}}=\left(\varrho_{R}, u_{R}, p_{R}\right) .
\end{aligned}
$$

Here we show the relations between primitive variables in $\Omega_{L} \cup \Omega_{H T L} \cup \Omega_{\star L}$ (across the left wave), and the set of relations for primitive variables in $\Omega_{\star R} \cup \Omega_{R} \cup \Omega_{H T R}$ (across the right wave).

\section{- 1-shock wave}

One of the possible wave patterns connecting $\Omega_{L}$ and $\Omega_{\star L}$ is a shock wave. Region $\Omega_{H T L}$ degenerates into single half-line. Primitive variables $\varrho, u, p$ "jump“ accross this wave. Inviscid shock jump relations can be derived, we call them Rankine-Hugoniot relations. These leads us to the following relations across the 1-shock wave (see [5], or [2, page 125])

$$
\begin{gathered}
u_{\star}=u_{L}-\left(p_{\star}-p_{L}\right)\left(\frac{\frac{2}{(\gamma+1) \varrho_{L}}}{p_{\star}+\frac{\gamma-1}{\gamma+1} p_{L}}\right)^{\frac{1}{2}}, \\
\varrho_{\star L}=\varrho_{L} \frac{(\gamma+1) p_{\star}+(\gamma-1) p_{L}}{(\gamma-1) p_{\star}+(\gamma+1) p_{L}}, \\
s_{1}=u_{L}-a_{L} \sqrt{\frac{\gamma+1}{2 \gamma} \frac{p_{\star}}{p_{L}}+\frac{\gamma-1}{2 \gamma}},
\end{gathered}
$$

where $a_{L}=\sqrt{\gamma \frac{p_{L}}{\rho_{L}}}$ is the speed of sound in the $\Omega_{L}, s_{1}$ denotes speed of the 1-shock wave. Half line $\frac{\tilde{x}_{1}}{t}=s_{1}$ shapes the boundary between $\Omega_{L}$ and $\Omega_{\star L}$. With the (13) known, (15),(16),(17) form the system of three equations for four unknowns $\varrho_{\star L}, p_{\star}, u_{\star}, s_{1}$. It is $u_{\star}<$ $u_{L}, p_{\star}>p_{L}, \varrho_{\star L}>\varrho_{L}$ across this wave, see [2, page 121-122]. Also the speed of sound and the temperature increases across this wave.

\section{- 1-rarefaction wave}

Another possible left wave pattern is rarefaction wave. It forms $\Omega_{H T L}$ region. Variables change smoothly within this wave. Across the 1-rarefaction wave $p_{\star} \leq p_{L}, u_{\star} \geq$ $u_{L}$. The following relations hold

$$
\begin{gathered}
u_{\star}=u_{L}+\frac{2}{\gamma-1} a_{L}\left[1-\left(\frac{p_{\star}}{p_{L}}\right)^{(\gamma-1) / 2 \gamma}\right], \\
\varrho_{\star L}=\varrho_{L}\left(\frac{p_{\star}}{p_{L}}\right)^{\frac{1}{\gamma}}, \\
s_{T L}=u_{\star}-a_{L}\left(\frac{p_{\star}}{p_{L}}\right)^{(\gamma-1) / 2 \gamma} .
\end{gathered}
$$

Here $s_{T L}$ is speed of the tail of the 1-rarefaction wave. From the speed of sound definition $a=\sqrt{\frac{\gamma p}{\varrho}}$ and (19) we get another useful relation across the 1-rarefaction wave

$$
a_{\star L}=a_{L}\left(\frac{p_{\star}}{p_{L}}\right)^{\frac{\gamma-1}{2 \gamma}} .
$$

It is $a_{\star L} \leq a_{L}$ for 1-rarefaction wave. Speed of the head of the rarefaction wave can be expressed $s_{H L}=u_{L}-a_{L}$. Half line $\frac{\tilde{x}_{1}}{t}=s_{T L}$ forms the boundary between $\Omega_{L}$ and $\Omega_{H T L}$. Primitive variables in $\Omega_{H T L}$ change continuously, see [2], (3.1.97), page 118. Pressure positivity for $p_{\star}$ in (18) gives the condition $u_{\star}<u_{L}+\frac{2}{\gamma-1} a_{L}$. 


\section{- 3-shock wave}

One of the possible right wave pattern is a 3 -shock wave. Region $\Omega_{H T R}$ degenerates into a single half-line. The primitive variables $\varrho, u, p$ are discontinues across this half line. Following relations for the primitive variables hold

$$
\begin{gathered}
u_{\star}=u_{R}+\left(p_{\star}-p_{R}\right)\left(\frac{\frac{2}{(\gamma+1) \varrho_{R}}}{p_{\star}+\frac{\gamma-1}{\gamma+1} p_{R}}\right)^{\frac{1}{2}}, \\
\varrho_{\star R}=\varrho_{R} \frac{\frac{p_{\star}}{p_{R}}+\frac{\gamma-1}{\gamma+1}}{\frac{\gamma-1}{\gamma+1} \frac{p_{\star}}{p_{R}}+1}, \\
s_{3}=u_{R}+a_{R} \sqrt{\frac{\gamma+1}{2 \gamma} \frac{p_{\star}}{p_{R}}+\frac{\gamma-1}{2 \gamma}} .
\end{gathered}
$$

Here $s_{3}$ denotes the velocity of the 3 -shock wave, $a_{R}=$ $\sqrt{\gamma \frac{p_{R}}{\rho_{R}}}$ is the speed of sound in the region $\Omega_{R}$. It is $p_{\star}>$ $p_{R}, u_{\star}>u_{R}$ across the 3 -shock wave.

\section{- 3-rarefaction wave}

Another possible wave pattern forming the region $\Omega_{H T R}$ is the rarefaction wave. Primitive variables change smoothly across this wave. It holds that

$$
\begin{gathered}
u_{\star}=u_{R}-\frac{2}{\gamma-1} a_{R}\left[1-\left(\frac{p_{\star}}{p_{R}}\right)^{(\gamma-1) / 2 \gamma}\right], \\
\varrho_{\star R}=\varrho_{R}\left(\frac{p_{\star}}{p_{R}}\right)^{\frac{1}{\gamma}}, \\
s_{T R}=u_{\star}+a_{R}\left(\frac{p_{\star}}{p_{R}}\right)^{(\gamma-1) / 2 \gamma} .
\end{gathered}
$$

In the last relation $s_{T R}$ denotes the speed of the tail of the 3-rarefaction wave. Half line $S_{H R}=\left\{\left(\tilde{x}_{1}, t\right) ; \tilde{x}_{1}=\right.$ $\left.s_{H R} t ; t>0\right\}$ is the boundary between $\Omega_{H T R}$ and $\Omega_{R}$, and half line $S_{T R}=\left\{\left(\tilde{x}_{1}, t\right) ; \tilde{x}_{1}=s_{T R} t ; t>0\right\}$ is the boundary between $\Omega_{\star R}$ and $\Omega_{H T R}$. The velocity $s_{H R}$ can be expressed

$$
s_{H R}=u_{R}+a_{R} .
$$

It is $p_{\star} \leq p_{R}, u_{\star} \leq u_{R}$ across the 3 -shock wave. Primitive variables in $\Omega_{H T R}$ change smoothly, see [2], [7].

Now, the combination of (15), (18), (22) and (25) gives the implicit equation for the unknown pressure $p_{\star}$, the analysis can be found in [2]. This is a nonlinear algebraic equation, and one cannot express the analytical solution of this problem in a closed form. The Newton method can be used to find the solution for the pressure $p_{\star}$. With the pressure $p_{\star}$ known, it is possible to use the equations (15)-(27) to compute the remaining unknowns in the solution of the Riemann problem (12),(13),(14).

\section{Boundary Condition for the Diffusible Barrier}

Here we present the diffusible barrier condition as a combination of Darcy's law with the additional innertial losses.

$$
\frac{\Delta p}{\Delta m}=-\left(\frac{\mu}{\alpha} U+C_{o} \frac{1}{2} R U^{2}\right), \text { where }
$$

$\Delta p=p_{2}-p_{1} \ldots$ pressure difference across the barrier

$\Delta m \ldots$ thickness of the barrier, example $\Delta m \in(0.01,0.05)$

$\mu$... dynamic viscosity

$\alpha$... permeability coefficient, example $\alpha=10^{-8}$

$C_{o} \ldots$ pressure gradient coefficient, example $C_{o}=10^{2}$

$R$... density at the barrier

$U$... velocity at the barrier

The equation (29) can be rewritten in the form

$$
R U^{2}+C U=d\left(p_{1}-p_{2}\right),
$$

here $C=\frac{2 \mu}{\alpha C_{o}}>0, d=\frac{2}{\Delta m C_{o}}>0,\left(p_{1}-p_{2}\right) \geq 0$. We are interested in the boundary values $R, U, P$ at the barrier. Further we require that the conservation laws (1D Euler equations) are satisfied in the close vicinity of the barrier

$\frac{\partial}{\partial t}\left(\begin{array}{c}\varrho \\ \varrho u \\ E\end{array}\right)+\frac{\partial}{\partial \tilde{x}_{1}}\left(\begin{array}{c}\varrho u \\ \varrho u^{2}+p \\ (E+p) u\end{array}\right)=0, \quad t \in(0, \infty), \tilde{x}_{1} \in(-\infty, \infty)$.

Here the axis $\tilde{x}_{1}$ is perpendicular to the barrier, $\varrho\left(\tilde{x}_{1}, t\right)$ denotes the density, $p\left(\tilde{x}_{1}, t\right)$ is the pressure, $u\left(\tilde{x}_{1}, t\right)$ is the velocity (with the direction perpendicular to the barrier), $E\left(\tilde{x}_{1}, t\right)$ denotes the total energy: $E=\varrho u^{2} / 2+p /(\gamma-1)$. The initial condition is formed by the two states near the barrier. Let us denote these states $\varrho_{1}, u_{1}, p_{1}$ and $\varrho_{2}, u_{2}, p_{2}$ :

$$
\begin{array}{ll}
\varrho\left(\tilde{x}_{1}, 0\right)=\varrho_{1} \text { for } \tilde{x}_{1}<0, & \varrho\left(\tilde{x}_{1}, 0\right)=\varrho_{2} \text { for } \tilde{x}_{1}>0, \\
u\left(\tilde{x}_{1}, 0\right)=u_{1} \text { for } \tilde{x}_{1}<0, & u\left(\tilde{x}_{1}, 0\right)=u_{2} \text { for } \tilde{x}_{1}>0, \\
p\left(\tilde{x}_{1}, 0\right)=p_{1} \text { for } \tilde{x}_{1}<0, & p\left(\tilde{x}_{1}, 0\right)=p_{2} \text { for } \tilde{x}_{1}>0 .
\end{array}
$$

In Section 4 we have shown the unique solution (entropy weak) of the Riemann problem (31),(32). Adding another condition (30) into this complete system doesn't make sense. Therefore we think of the barrier problem as of two boundary problems with the two particular solutions (different in general).

- Problem 1. Find the solution $R, U, P$ of the system (31), equipped with the one-side initial condition $\varrho_{1}, u_{1}, p_{1}$ and the boundary condition (30).

- Problem 2. Find the solution $R, U, P$ of the system (31), equipped with the one-side initial condition $\varrho_{2}, u_{2}, p_{2}$ and the boundary condition (30).

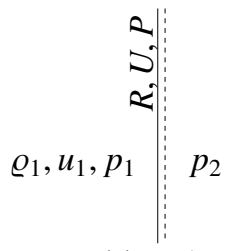

Problem 1.

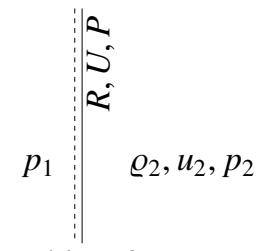

Problem 2.
Further we will show the solution of these two problems.

Remark 1. The condition (30) is not rotationally invariant! Let us think of a Problem 1. with the given initial state $\varrho_{1}, u_{1}, p_{1}=\tilde{\varrho}, \tilde{u}, \tilde{p}$ and $p_{2}=p_{X}$, and let $R_{1}, U_{1}, P_{1}$ be the solution satisfying $R_{1} U_{1}^{2}+C U_{1}=d\left(\tilde{p}-p_{X}\right)$. This situation is symmetric to the Problem 2. with the given $\varrho_{2}, u_{2}, p_{2}=$ $\tilde{\varrho},-\tilde{u}, \tilde{p}$ and $p_{1}=p_{X}$. Let $R_{2}, U_{2}, P_{2}$ be the solution of such problem satisfying $R_{2} U_{2}^{2}+C U_{2}=d\left(\tilde{p}-p_{X}\right)$. This solution should be symmetric (to the solution of the previous Problem 1.): $R_{2}, U_{2}, P_{2}=R_{1},-U_{1}, P_{1}$. Then $R_{2} U_{2}^{2}+C U_{2}=$ $R_{1} U_{1}^{2}-C U_{1}=d\left(\tilde{p}-p_{X}\right)$, which is possible only if $p_{1}=p_{2}$. 
Remark 2. Non-uniqueness of the solution: There is a possibility of multiple solutions to the Problem 1 . Let $u_{1}>0$, and let us choose the velocity $U<0$ arbitrarily. Then there is a solution with the 1-shock wave, and the sought boundary state is $R, U, P$ where

$P=\frac{2 p_{1}+\frac{\gamma+1}{2} \varrho_{L}\left(u_{1}-u_{\star}\right)^{2}+\left(u_{1}-u_{\star}\right) \sqrt{4 \varrho_{1} \gamma p_{1}+\varrho_{1}^{2}\left(\frac{\gamma+1}{2}\right)^{2}\left(u_{1}-u_{\star}\right)^{2}}}{2}$.

This relation, shown in [3], is rewritten relation (15). Further (due to the possible density jump across the contact discontinuity) we choose density $R=\left(d\left(p_{1}-p_{2}\right)-C U\right) / U^{2}$.

As a consequence of the remarks above, we limit the Problem 1. in order to achieve the uniqueness of the solution. We suppose the initial velocity $u_{1} \geq 0$, and we seek the solution with $U>0$. According to the analysis of the Riemann problem we use the equations for the left wave and the complementary condition (30). There are two possible patterns of the left wave.

\section{1-shock wave: $u_{1} \geq U \geq 0$}

In this case we use the formulae (15),(16) for the shock wave, valid for $P \geq p_{1}$.

$$
\begin{gathered}
U(P)=u_{1}-\frac{P-p_{1}}{a_{1}(P)}, \quad a_{1}(P)=\sqrt{\varrho_{1}\left(\frac{\gamma+1}{2} P+\frac{\gamma-1}{2} p_{1}\right)}, \\
R(P)=\frac{a_{1}(P) \varrho_{1}}{a_{1}(P)-\varrho_{1}\left(u_{1}-U\right)} .
\end{gathered}
$$

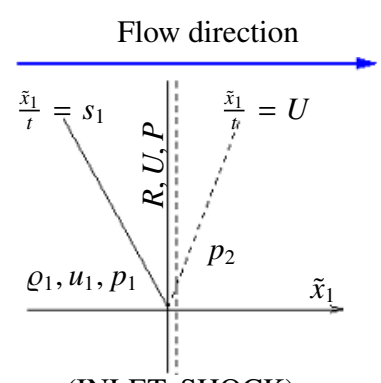

$$
\begin{aligned}
& R U^{2}+C U=d\left(p_{1}-p_{2}\right) \\
& \downarrow \begin{array}{c}
U(P)=u_{1}-\frac{P-p_{1}}{a_{1}(P)} \\
R(P)=\frac{a_{1}(P) \varrho_{1}}{a_{1}(P)-\varrho_{1}\left(u_{1}-U\right)} \\
l(P)=R U^{2}+C U
\end{array} \\
& \text { solve } l(P)=d\left(p_{1}-p_{2}\right)
\end{aligned}
$$

(INLET, SHOCK)

Fig. 4. Diffusible barrier, $R, U, P$ is the sought state at the boundary, $\varrho_{1}, u_{1}, p_{1}, p_{2}$ present the given initial condition, situation with the shock wave (speed $s_{1}<0$ ).

The velocity of the shock wave $s_{1}=u_{1}-a_{1}(P) / \varrho_{1}$, see (17). We require the boundary value condition (30) to be applicable. So it must be $s_{1} \leq 0$. Using (17), this is satisfied only if

$$
P \geq p_{1}\left[1+\frac{2 \gamma}{\gamma+1}\left(M_{1}^{2}-1\right)\right], \quad M_{1}^{2}=u_{1}^{2} / c_{1}^{2},
$$

where $c_{1}=\sqrt{\gamma p_{1} / \varrho_{1}}$ is the speed of sound, and $M$ denotes the Mach number. If $u_{1}<c_{1}$, then $s_{1} \leq 0$ for every $P \geq p_{1}$. The function $U(P)$ is decreasing, let us denote $P^{o}$ the pressure satisfying $U\left(P^{o}\right)=0$, it is $P^{o} \geq p_{1}$. Now we define a function $l(P)=R(P) U^{2}(P)+C U(P)$ in the interval $\left\langle p_{1}, P^{o}\right\rangle$, using the formula (33),(34). We have got

$$
l(P)=l_{a}(P)+l_{b}(P), \text { with }
$$

$$
l_{a}(P)=\frac{\left(a_{1}(P) u_{1}-\left(P-p_{1}\right)\right)^{2}}{P(\gamma-1) / 2+p_{1}(\gamma+1) / 2}, \quad l_{b}=C\left(u_{1}-\frac{P-p_{1}}{a_{1}(P)}\right)
$$

where $l\left(p_{1}\right)=\varrho_{1} u_{1}^{2}+C u_{1}, l\left(P^{o}\right)=0$. We want to prove that the function $l(P)$ is monotonous in the interval $\left\langle p_{1}, P^{o}\right\rangle$. The function $l_{b}(P)$ is decreasing. We want to prove, that the function $l_{a}(P)$ has the same property in $\left\langle p_{1}, P^{o}\right\rangle$ :

It is difficult to guess the sign of the first derivative of this function except

$$
l_{a}^{\prime}\left(p_{1}\right)=\frac{u_{1} \varrho_{1}}{\gamma p_{1}}\left(u_{1}-2 c_{1}\right) \text { and } l_{a}^{\prime}\left(P^{o}\right)=0 .
$$

The added inequality $u_{1}<c_{1}$ implies $l_{a}^{\prime}\left(p_{1}\right)<0$. To study the monotony of $l_{a}(P)$ we use the following substitutions

$$
h^{2}(P)=l_{a}(P), \quad l_{a}^{\prime}(P)=2 h(P) h^{\prime}(P),
$$

where $h(P)>0$ in $\left\langle p_{1}, P^{o}\right)$, and $h\left(P^{o}\right)=0$. We use following trick to find the sign of $h^{\prime}(P)$. We use the next substitution to the formulae for $h^{\prime}(P)$

$$
m(P)=4 h^{\prime}(P) a_{1}(P) \sqrt{\frac{\gamma-1}{2} P+\frac{\gamma+1}{2} p_{1}} .
$$

It yields

$$
m(P)=\frac{2 \varrho_{1} u_{1} p_{1} \gamma-a_{1}(P)\left[(\gamma-1) P+(3 \gamma+1) p_{1}\right]}{P(\gamma-1) / 2+p_{1}(\gamma+1) / 2} \text { in }\left\langle p_{1}, P^{o}\right\rangle .
$$

Now we shall ask for a root of the equation $m(P)=0$ in the interval $\left\langle p_{1}, P^{o}\right\rangle$. So we must solve the equation

$$
a_{1}(P)=\frac{2 \varrho_{1} u_{1} p_{1} \gamma}{(\gamma-1) P+(3 \gamma+1) p_{1}}
$$

We know that $m\left(p_{1}\right)=2 \varrho_{1} p_{1} \gamma\left(u_{1}-2 c_{1}\right)<0$ and the right-hand side of equation (36) is decreasing and the left-hand side of (36) is increasing function. So we have proved that there is no root of equation (36) in $\left\langle p_{1}, P^{o}\right\rangle$. Hence, $l_{a}(P)$ is decreasing in $\left\langle p_{1}, P^{o}\right\rangle$.

1-rarefaction (expansion) wave: $u_{1} \leq U<u_{1}+\frac{2}{\gamma-1} c_{1}$ In this case we use the formulas for the 1-rarefaction wave (18), (19). Using these, the relation for the density $R$ can be rewritten

$$
R=\varrho_{1}\left(1-\frac{\left(U-u_{1}\right)(\gamma-1)}{2 c_{1}}\right)^{2 /(\gamma-1)}
$$

Let us define the function $s(U)=U^{2} R(U)+C U$ dependent on the velocity $U$ :

$$
s(U)=U^{2} \varrho_{1}\left(1-\frac{\left(U-u_{1}\right)(\gamma-1)}{2 c_{1}}\right)^{2 /(\gamma-1)}+C U .
$$

It is $s\left(u_{1}\right)=\varrho_{1} u_{1}^{2}+C u_{1}, \quad s\left(u_{1}+\frac{2}{\gamma-1} c_{1}\right)=C\left(u_{1}+\frac{2}{\gamma-1} c_{1}\right)$. The first derivative is

$$
\begin{array}{r}
s^{\prime}(U)=2 \varrho_{1} U\left(1-\frac{\left(U-u_{1}\right)(\gamma-1)}{2 c_{1}}\right)^{2 /(\gamma-1)} . \\
\cdot\left[1-\frac{U}{2 c_{1}}\left(1-\frac{\left(U-u_{1}\right)(\gamma-1)}{2 c_{1}}\right)^{-1}\right]+C .
\end{array}
$$

We assume $C>0$. It is $s^{\prime}(U)>0$ for $U \in\langle 0, \infty) \cap\left\langle u_{1}, u_{1}+\right.$ $\left.\frac{2}{\gamma-1} c_{1}\right) \cap\left\langle u_{1}, \frac{2 c_{1}+(\gamma-1) u_{1}}{\gamma}\right)$. In order to apply the boundary value condition (30) it is necessary to study the velocities 


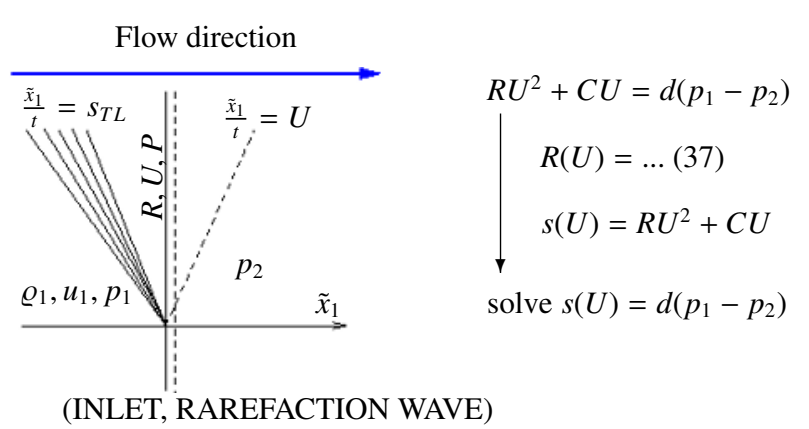

Fig. 5. Diffusible barrier, $R, U, P$ is the sought state at the boundary, $\varrho_{1}, u_{1}, p_{1}, p_{2}$ present the given initial condition, situation with the 1-rarefaction wave.

of the front and the back of the rarefaction wave. The speed of the tail of the rarefaction wave (20) can be written as

$$
s_{T L}=U-c_{1}^{*}, \text { where } c_{1}^{*}=c_{1}+\frac{\gamma-1}{2}\left(u_{1}-U\right) .
$$

We need $s_{T L} \leq 0$. So we must add the other condition

$$
u_{1} \leq U \leq \frac{2 c_{1}+(\gamma-1) u_{1}}{\gamma+1} .
$$

Therefore we restrict the former domain of the function $s(U)$ to the interval $\left\langle u_{1}, \frac{2 c_{1}+(\gamma-1) u_{1}}{\gamma+1}\right\rangle$.

\section{Solution of the Problem 1. (INLET)}

Now we can resume how to solve this boundary value problem. First of all we must mention the conditions of a solution by given $p_{1}, \varrho_{1}, u_{1}$, and (30). The first condition is $u_{1}<c_{1}$, and the second one is

$$
d\left(p_{1}-p_{2}\right) \in\left\langle 0, s\left(\frac{2 c_{1}+(\gamma-1) u_{1}}{\gamma+1}\right)\right\rangle
$$

- If $d\left(p_{1}-p_{2}\right) \in\left\langle 0, \varrho_{1} u_{1}^{2}+C u_{1}\right\rangle$, then we solve equation $l(P)=d\left(p_{1}-p_{2}\right)$. Once the pressure $\mathrm{P}$ is known, the velocity $U$ and the density $R$ is computed using (33), (34).

- If $d\left(p_{1}-p_{2}\right) \in\left\langle\varrho_{1} u_{1}^{2}+C u_{1}, s\left(\frac{2 c_{1}+(\gamma-1) u_{1}}{\gamma+1}\right)\right\rangle$, then the equation $s(U)=d\left(p_{1}-p_{2}\right)$ is solved for the unknown velocity $U$. The sought pressure $P$ and the density $R$ is then computed using (18), (19).

The results have been proved to be unique.

Now we must study the boundary value problem for outlet from the barrier. The positive orientation is out of the barrier. Let us suppose that $u_{2} \geq 0$. The state values $\varrho_{2}, u_{2}, p_{2}$ are known on the back of this barrier, and $p_{1}$ is the known pressure on the front side. Again, we consider two possibilities (based on the Section 4): the 3-shock wave and the 3-rarefation wave.

3-shock: $U \geq u_{2}$

Here we work with the formulas (22),(23) for the 3-shock wave, valid for $U \geq u_{2}, P \geq p_{2}$.

$$
U(P)=u_{2}+\frac{P-p_{2}}{a_{2}(P)}, \quad a_{2}(P)=\sqrt{\varrho_{2}\left(\frac{\gamma+1}{2} P+\frac{\gamma-1}{2} p_{2}\right)}
$$

$$
R(P)=\frac{a_{2}(P) \varrho_{2}}{a_{2}(P)+\varrho_{2}\left(u_{2}-U\right)}=\varrho_{R} \frac{(\gamma+1) P+(\gamma-1) p_{R}}{(\gamma-1) P+(\gamma+1) p_{R}} .
$$

The functions $R(P)$ and $U(P)$, defined above, are increasing in the interval $\left\langle p_{2}, \infty\right)$. It is $R>\varrho_{2}$.

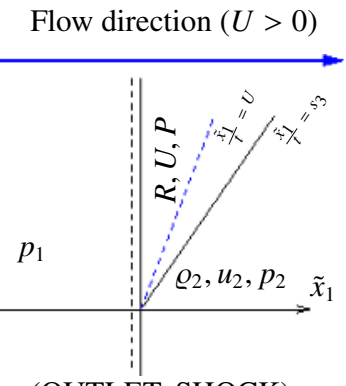

(OUTLET, SHOCK)

$$
\begin{aligned}
& R U^{2}+C U=d\left(p_{1}-p_{2}\right) \\
& \downarrow \begin{array}{c}
U(P)=u_{2}+\frac{P-p_{2}}{a_{2}(P)} \\
R(P)=\frac{a_{2}(P) \varrho_{2}}{a_{2}(P)+\varrho_{2}\left(u_{2}-U\right)} \\
l(P)=R U^{2}+C U
\end{array} \\
& \text { solve } l(P)=d\left(p_{1}-p_{2}\right)
\end{aligned}
$$

Fig. 6. Diffusible barrier, $R, U, P$ is the sought state at the boundary, $\varrho_{2}, u_{2}, p_{2}, p_{1}$ present the given initial condition, situation with the shock wave (speed $s_{3}>0$ ).

Now we define the function $l(P)=R(P) U^{2}(P)+C U(P)$, using the formulas (40),(41). It is

$$
\begin{gathered}
l(P)=l_{a}(P)+l_{b}(P), \text { with } \\
l_{a}(P)=\frac{\left(a_{2}(P) u_{2}+\left(P-p_{2}\right)\right)^{2}}{P(\gamma-1) / 2+p_{2}(\gamma+1) / 2}, \quad l_{b}=C\left(u_{2}+\frac{P-p_{2}}{a_{2}(P)}\right) .
\end{gathered}
$$

The function $l_{a}(P)$ is increasing in the interval $\left\langle p_{2}, \infty\right)$. Further the function $l_{b}(P)$ is increasing and the same property has the function $l(P)$. It is $l\left(p_{2}\right)=\varrho_{2} u_{2}^{2}+C u_{2}$. We require the boundary value condition (30) to be applicable at the boundary. This can be satisfied only if $s_{3} \geq 0$, which comes from the analysis in the Section 4. Using (24), it is $s_{3} \geq 0$ only if

$$
P \geq p_{2}\left[1+\frac{2 \gamma}{\gamma+1}\left(M_{2}^{2}-1\right)\right], \quad M_{2}^{2}=u_{2}^{2} / c_{2}^{2},
$$

where $c_{2}=\sqrt{\gamma p_{2} / \varrho_{2}}$ is the speed of sound, and $M$ denotes the Mach number. If $u_{2} \geq 0$, then (43) is satisfied.

\section{3-rarefaction (expansion): $U \leq u_{2}$}

Here we work with the formulas (25),(26) for the 3-rarefaction wave, valid for $u_{2}-\frac{2 c_{2}}{\gamma-1} \leq U \leq u_{2}, P \leq p_{2}$. The density $R$ can be written as a function of the velocity $U$

$$
R(U)=\varrho_{2}\left(1+\frac{\left(U-u_{2}\right)(\gamma-1)}{2 c_{2}}\right)^{2 /(\gamma-1)} .
$$

Now we define the function $s(U)=U^{2} R(U)+C U$ dependent on a velocity $U, U \in\left\langle 0, u_{2}\right\rangle$ :

$$
s(U)=\varrho_{2} U^{2}\left(1+\frac{\left(U-u_{2}\right)(\gamma-1)}{2 c_{2}}\right)^{2 /(\gamma-1)}+C U .
$$

The function $s(U)$ is increasing in the interval $\left\langle 0, u_{2}\right\rangle$. It is $s(0)=0, \quad s\left(u_{2}\right)=\varrho_{2} u_{2}^{2}+C u_{2}$.

\section{Solution of the Problem 2. (OUTLET)}

Now we can resume how to solve the boundary value problem for the back side of the barrier. We suppose zero density jump across the middle wave. 


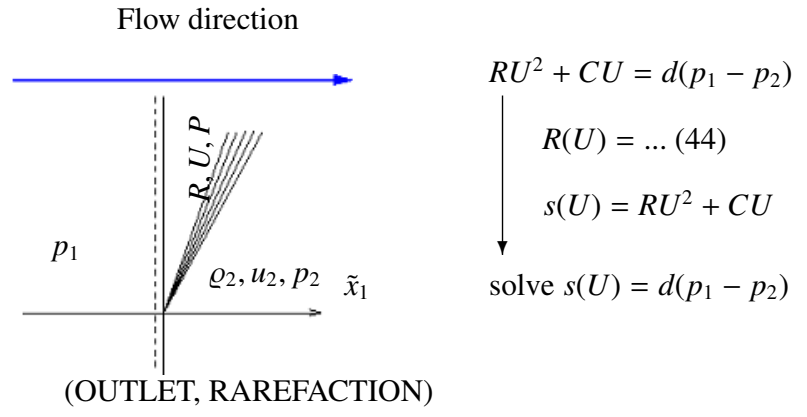

Fig. 7. Diffusible barrier, $R, U, P$ is the sought state at the boundary, $\varrho_{2}, u_{2}, p_{2}, p_{1}$ present the given initial condition, situation with the rarefaction wave.

- If $d\left(p_{1}-p_{2}\right) \in\left\langle 0, \varrho_{2} u_{2}^{2}+C u_{2}\right\rangle$, then we solve the equation $s(U)=d\left(p_{1}-p_{2}\right)$, with $s(U)$ defined in (45). Then the sought pressure $P$ and the density $R$ is, accordingly to (25), (26).

- For $d\left(p_{1}-p_{2}\right)>\varrho_{2} u_{2}^{2}+C u_{2}$ we solve the equation $l(P)=d\left(p_{1}-p_{2}\right)$, where $l(P)$ is defined in (42). Then the velocity $U$ and the density $R$ is computed using (40), (41).

The results have been proved to be unique.

\section{Examples}

Here we show the computational results of the compressible gas flow, obtained with the own-developed codes (the RANS with the k-w model of turbulence). The gas constants for the air are $\gamma=1.4$ and $R=287.04[\mathrm{~J} / \mathrm{kgK}]$. We use the viscosity coefficient (depending on the local temperature) computed with the use of the Sutherland's law $\mu=\mu_{\text {ref }}\left(\frac{T}{T_{r e f}}\right)^{3 / 2} \frac{T_{r e f}+S}{T+S}$, with $S=110.4[\mathrm{~K}], T_{r e f}=$ $273.15[\mathrm{~K}], \mu_{r e f}=1.716 e-05[\mathrm{~Pa} \mathrm{~s}]$, and the heat conduction coefficient $k=0.0211$. The Figs. 9. - 11. show the computed result of the flow through the diffusible barrier enclosed in the channel. The following setup was used:

- geometry - the given geometry is shown in Fig. 8.

- initial condition - constant state in the whole domain, $T_{o}=273.15[\mathrm{~K}], \boldsymbol{v}=(0,0), p^{o}=101325[\mathrm{~Pa}]$.

- inlet boundary condition (left) - total quantities and velocity direction. This boundary condition was described in [4], [3], total pressure $p_{o}=101325[\mathrm{~Pa}$ ], total temperature $T_{o}=273.15[K]$, and zero tangential velocity.

- outlet boundary condition (right) - preference of the pressure, see [3]. Given pressure $p_{\star}=95009.2[\mathrm{~Pa}]$ and averaging technique was used.

- wall boundary condition (up, bottom) - preference of the velocity, see [3]. Zero velocity at the wall was given together with the wall temperature $T_{W A L L}=273.15[\mathrm{~K}]$.

- diffusible barrier - boundary condition described in Section 5. with constants $C_{0}=10^{2}, \Delta m=0.01, \alpha=10^{-8}$.

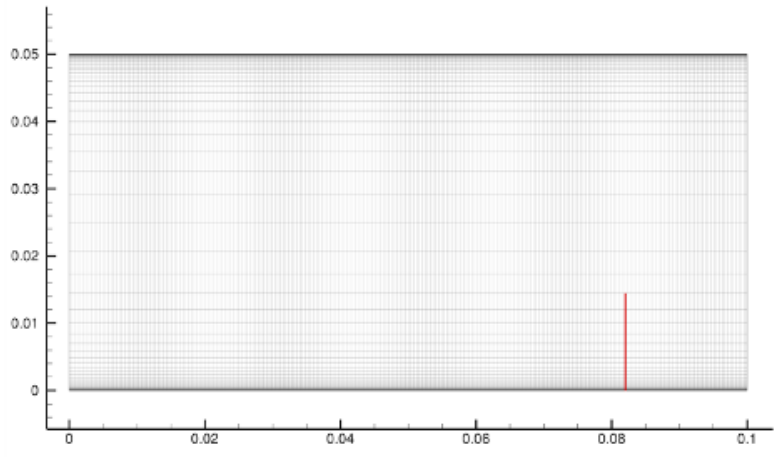

Fig. 8. The diffusible barrier, computational mesh consisted of $244 \times 86$ elements. Red line represents the diffusible barrier.

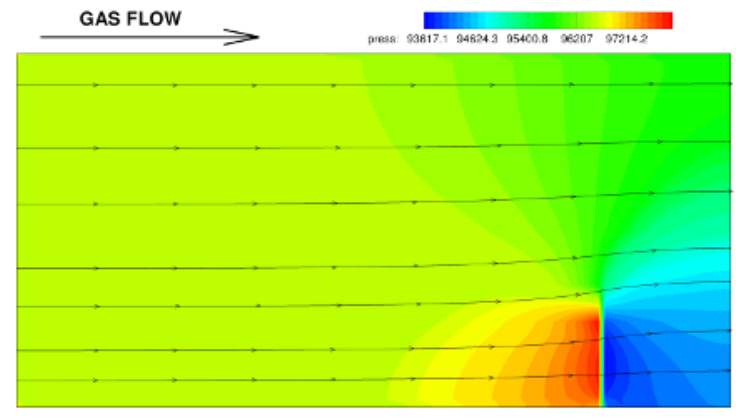

Fig. 9. Compressible gas flow through the diffusible barrier, pressure isolines and chosen velocity streamlines.



Fig. 10. Compressible gas flow through the diffusible barrier, density isolines.

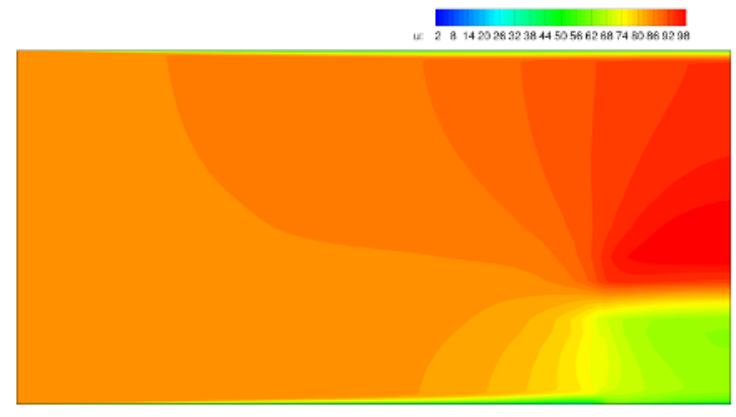

Fig. 11. Compressible gas flow through the diffusible barrier, $x-$ velocity component isolines.

Another computational example shows the compressible gas flow through the two consequent barriers, see Fig 12.-15. 


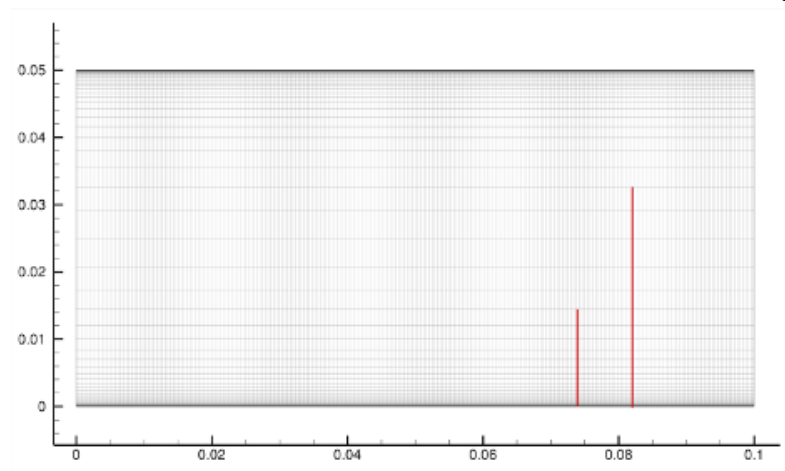

Fig. 12. The diffusible barrier, computational mesh consisted of $244 \times 86$ elements. Red lines represent the diffusible barriers.

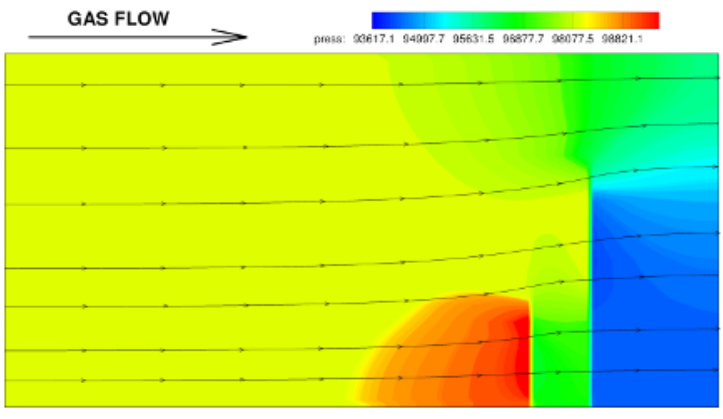

Fig. 13. Two consequent diffusible barriers, pressure isolines.

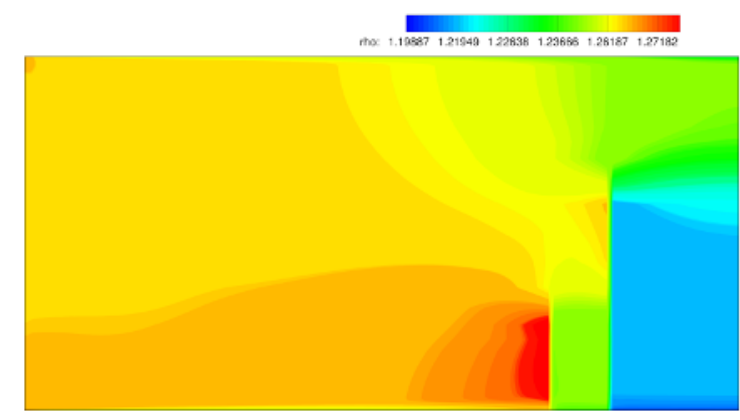

Fig. 14. Two consequent diffusible barriers, density isolines.

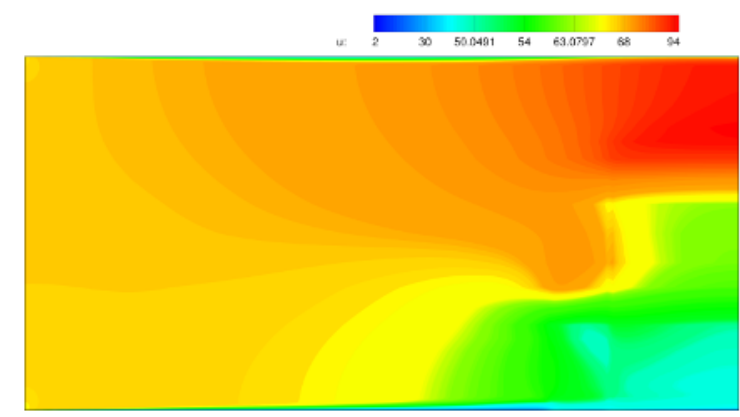

Fig. 15. Two consequent diffusible barriers, x-velocity component isolines.

\section{Conclusion}

This paper shows the analysis of the boundary condition for the diffusible barrier. This boundary condition is then used in the finite volume method for the simulation of the compressible gas flow through the diffusible barrier. The numerical examples of the two-dimensional flow around diffusible barrier were presented.

\section{References}

1. M. Feistauer. Numerical methods for compressible flow. In J. Neustupa and P. Penel, editors, Mathematical Fluid Mechanics. Recent Results and Open Questions. Birkhäuser, Basel, 2001.

2. M. Feistauer, J. Felcman, and I. Straškraba. Mathematical and Computational Methods for Compressible Flow. Oxford University Press, Oxford, 2003.

3. M. Kyncl. Numerical solution of the three-dimensional compressible flow. Master's thesis, Prague, 2011. Doctoral Thesis.

4. M. Kyncl and J. Pelant. Applications of the NavierStokes equations for $3 \mathrm{~d}$ viscous laminar flow for symmetric inlet and outlet parts of turbine engines with the use of various boundary conditions. Technical report R3998, VZLÚ, Beranových 130, Prague, 2006.

5. M. Kyncl and J. Pelant. Applications of the NavierStokes equations for viscous turbulent flow on steady or moving grids with the (k,omega) turbulent model. Technical report R4153, VZLÚ, Beranových 130, Prague, 2007.

6. J. Pelant. Arti reports VZLÚ, z-65, z-67 to z-73. Prague, 1996-2000.

7. E. F. Toro. Riemann Solvers and Numerical Methods for Fluid Dynamics. Springer, Berlin, 1997. 